\title{
A CONSTRUCTION OF FINITE AND $\sigma$-FINITE INVARIANT MEASURES IN MEASURE SPACES
}

\author{
YOSHIHIRO KUBOKAWA
}

(Communicated by R. Daniel Mauldin)

\begin{abstract}
Let $T$ be a bijective nonsingular transformation on a finite measure space. We shall first construct a $\sigma$-finite and finite invariant measure by a unified method which is valid for both cases. Secondly we shall give another construction of a finite invariant measure. We shall also give a new necessary and sufficient condition of a unified form for the existence of $\sigma$-finite and finite invariant measures. Further, we shall discuss in detail ergodic transformations.
\end{abstract}

1. Introduction. Let $T$ be a bijective nonsingular transformation on a finite measure space $(X, \mathcal{F}, m)$. This means that $T$ is a bimeasurable bijection and $m\left(T^{-1} E\right)=m(T E)=0$ for any measurable set $E$ with $m(E)=0$. Let $\mu$ and $\nu$ be $\sigma$-finite measures on $(X, \mathcal{F}) . \mu$ is said to be absolutely continuous with respect to $\nu$ if $\mu(E)=0$ for any measurable set $E$ with $\nu(E)=0$. In this case we write simply $\mu \ll \nu$. If $\mu \ll \nu$ and $\nu \ll \mu$, then we say that $\mu$ and $\nu$ are equivalent or $\mu$ is equivalent to $\nu$ and write simply $\mu \sim \nu$. A measure $\mu$ is said to be invariant if $\mu\left(T^{-1} E\right)=\mu(T E)=\mu(E)$ for any measurable set $E$. A measurable set $E$ is invariant if $T E=E$. A transformation $T$ is ergodic if $m(E)=0$ or $m\left(E^{c}\right)=0$ for any invariant measurable set $E$. Construction of finite invariant measures were studied by Hopf [5], Dowker [2], Calderón [1] and Hajian and Kakutani [3], while Halmos [4] studied $\sigma$-finite invariant measures. We shall present a unified construction which is useful for both cases at the same time. Although the idea can be found already in Hopf [5] and Halmos [4] we shall use general measure theory and give a more systematic treatment. We put $F^{*}=\bigcup_{n=-\infty}^{\infty} T^{n} F$ for any measurable set $F$. We fix a measurable set $E$. Putting $A^{\prime}=A \cap E^{*}$ for any measurable set $A$, we define $m_{E}$ by

$$
\begin{aligned}
m_{E}(A)=\inf \left\{\sum_{n=-\infty}^{\infty} m\left(T^{n} A_{n}\right), A_{n} \in \mathcal{F}(n=0, \pm 1, \pm 2, \ldots),\right. \\
\left.A^{\prime} \subset \bigcup_{n=-\infty}^{\infty} A_{n} \text { and } \bigcup_{n=-\infty}^{\infty} T^{n} A_{n} \subset E\right\} .
\end{aligned}
$$

Then $m_{E}$ is a $\sigma$-finite invariant measure with $m_{E} \ll m$ (Theorem 1). For any $\sigma$-finite invariant measure $\mu$ with $\mu \ll m$ there exists a measurable set $E$ with $\mu \sim m_{E}$ (Theorem 2). Further, if $\mu$ is finite we can find a measurable set $E$ with $m_{E}(X)<\infty$. From this we can give a necessary and sufficient condition

Received by the editors November 3, 1986.

1980 Mathematics Subject Classification (1985 Revision). Primary 28D05.

Key words and phrases. Construction of invariant measure, nonsingular transformation, $\sigma$-finite invariant measure, finite measure space. 
for the existence of a $\sigma$-finite or finite invariant measure which is equivalent to $m$ (Corollary 1 or 2). In Theorem 4 we shall give a necessary and sufficient condition for the existence of a $\sigma$-finite invariant measure which is equivalent to the given measure $m$ in case $T$ is ergodic. We shall discuss ergodic transformations in detail in Theorems 5 and 6 . Finally we shall generalize the well-known fact that an invariant measure exists uniquely in case $T$ is ergodic. Using this idea we shall give another construction of a finite invariant measure.

2. Construction and existence theorems. We first give a unified construction of invariant measures applicable to finite as well as $\sigma$-finite cases. We also give a criterion for the existence of an invariant measure which is equivalent to $m$.

THEOREM 1. For any measurable set $E, m_{E}$ is a $\sigma$-finite invariant measure with $m_{E} \ll m$.

ProOF. We define a set function $\mu_{E}$ by

$$
\begin{array}{r}
\mu_{E}(A)=\inf \left\{\sum_{n=-\infty}^{\infty} m\left(T^{n} A_{n}\right): A^{\prime} \subset \bigcup_{n=-\infty}^{\infty} A_{n}, T^{n} A_{n} \subset E\right. \\
\left.\quad \text { and } A_{n} \in \mathcal{F}(n=0, \pm 1, \pm 2, \ldots)\right\}
\end{array}
$$

for any subset $A$ of $X$. Then $\mu_{E}$ is an outer measure which satisfies $\mu_{E}(T A)=$ $\mu_{E}(A)$ for any subset $A$ of $X$. It is easy to check that any $\mathcal{F}$-measurable set is $\mu_{E}$-measurable. Therefore we can define a measure on $(X, \mathcal{F})$ by

$$
\begin{aligned}
m_{E}(A)=\inf \left\{\sum_{n=-\infty}^{\infty} m\left(T^{n} A_{n}\right): A^{\prime}=\bigcup_{n=-\infty}^{\infty} A_{n}, T^{n} A_{n} \subset E\right. \\
\left.\quad \text { and } A_{n} \in \mathcal{F}(n=0, \pm 1, \pm 2, \ldots)\right\}
\end{aligned}
$$

for any $A \in \mathcal{F}$. The nonsingularity of $T$ implies $m_{E} \ll m . m_{E}$ is $\sigma$-finite since $m_{E}$ is invariant, $m_{E}(E)<\infty$ and $m_{E}\left(E^{* c}\right)=0$.

THEOREM 2. Let $\mu$ be a $\sigma$-finite invariant measure with $\mu \ll m$. Then there exists a measurable set $E$ with $\mu \sim m_{E}$.

COROLlaRY 1. There exists a $\sigma$-finite invariant measure $\mu$ with $\mu \sim m$ if and only if there exists a measurable set $E$ with $m_{E} \gg m$.

Proof of TheOREM 2. Let $\alpha=\sup \{m(A): \mu(A)=0\}$. Then there exists an invariant measurable set $A$ such that $m(A)=\alpha$ and $\mu(A)=0$. Restricting the measures $\mu$ and $m$ to the invariant measurable set $A^{c}$ we have $\left.\left.\mu\right|_{A^{c}} \sim m\right|_{A^{c}}$. Therefore we need only to prove that there exists a measurable set $E$ with $m_{E} \sim m$ if there exists a $\sigma$-finite invariant measure $\mu$ with $\mu \sim m$. By Theorem $1 m_{E} \ll m$ for any measurable set $E$. We now show that there exists a measurable set $E$ with $m_{E} \gg m$. For the proof we need the following.

LEMMA. Let $\mu$ be a $\sigma$-finite invariant measure with $m \ll \mu$ and $f$ a RadonNikodým derivative $d m / d \mu$. We put

$$
E=\{x: 0<a \leq f(x) \leq b<\infty\} .
$$


Then for any measurable set $A$ we have

$$
a \mu\left(A \cap E^{*}\right) \leq m_{E}(A) \leq b \mu\left(A \cap E^{*}\right) .
$$

ProOF. By assumption for any measurable set $F$ we have $m(F)=\int_{F} f d \mu$ and

$$
\begin{aligned}
m_{E}(A)=\inf \left\{\sum_{n=-\infty}^{\infty} m\left(T^{n} A_{n}\right): A^{\prime} \subset \bigcup_{n=-\infty}^{\infty} A_{n}, T^{n} A_{n} \subset E\right. \\
\left.\quad \text { and } A_{n} \in \mathcal{F}(n=0, \pm 1, \pm 2, \ldots)\right\} .
\end{aligned}
$$

Since $A^{\prime}$ is also measurable, we may assume that the infimum runs over $A_{n}$ 's with $A^{\prime}=\bigcup_{n=-\infty}^{\infty} A_{n}$ (disjoint). We have

$$
\mu\left(A^{\prime}\right)=\sum_{n=-\infty}^{\infty} \mu\left(A_{n}\right) \quad \text { and } m\left(T^{n} A_{n}\right)=\int_{T^{n} A_{n}} f d \mu \quad(n=0, \pm 1, \pm 2, \ldots) .
$$

By assumption

$$
a \mu\left(T^{n} A_{n}\right) \leq \int_{T^{n} A_{n}} f d \mu \leq b \mu\left(T^{n} A_{n}\right)
$$

Since $\mu$ is invariant, we have

$$
a \mu\left(A_{n}\right) \leq m\left(T^{n} A_{n}\right) \leq b \mu\left(A_{n}\right) .
$$

We get the lemma by taking the infimum of the summation over $n$.

We now go back to the proof of Theorem 2 using the same notation as in the Lemma. $f(x)>0$ a.e., since $\mu \sim m$. We put

$$
\begin{gathered}
X_{n}=\{x: 1 /(n+1) \leq f(x) \leq n+1\} \quad \text { for } n=1,2, \ldots, \\
Y_{1}=X_{1} \quad \text { and } \quad Y_{n}=X_{n}-\sum_{i=1}^{n-1} Y_{i}^{*} \quad \text { for } n \geq 2 .
\end{gathered}
$$

Then $Y_{i}^{*} \cap Y_{j}^{*}=\varnothing$ for $i \neq j$ and $m\left(X-\bigcup_{n=1}^{\infty} Y_{n}^{*}\right)=0$. Putting $E=\bigcup_{n=1}^{\infty} Y_{n}$ we obtain a measure $m_{E}$ with $m \ll m_{E}$. Indeed if $m_{E}(A)=0$, then $m_{E}\left(A \cap Y_{n}^{*}\right)=0$ for any positive integer $n$. On the other hand, the definition of $m_{E}$ and $Y_{n}$, and $E \cap Y_{n}^{*}=Y_{n}$ imply

$$
m_{E}\left(A \cap Y_{n}^{*}\right)=m_{Y_{n}}\left(A \cap Y_{n}^{*}\right) .
$$

By the Lemma we have

$$
(1 /(n+1)) \mu\left(A \cap Y_{n}^{*}\right) \leq m_{E}\left(A \cap Y_{n}^{*}\right) \leq(n+1) \mu\left(A \cap Y_{n}^{*}\right) .
$$

This implies $\mu\left(A \cap Y_{n}^{*}\right)=0$ and therefore $m\left(A \cap Y_{n}^{*}\right)=0$ for $n=1,2,3, \ldots$ Thus we obtain $m(A)=0$.

THEOREM 3. Let $\mu$ be a finite invariant measure with $\mu \ll m$. Then there exists a measurable set $E$ such that $\mu \sim m_{E}$ and $m_{E}(X)<\infty$.

COROLLARY 2. There exists a finite invariant measure $\mu$ with $\mu \sim m$ if and only if there exists a measurable set $E$ such that $m \ll m_{E}$ and $m_{E}(X)<\infty$. In this case for any $\varepsilon>0$ we can find a measurable set $E$ with $m_{E}(X)<m(X)+\varepsilon$.

PROOF OF THEOREM 3. As in the case of Theorem 2, the proof of Theorem 3 is reduced to the case where there exists a finite invariant measure $\mu$ with $\mu \sim m$. 
We need only to prove that there exists a measurable set $E$ with $m_{E} \gg m$ and $m_{E}(X)<\infty$. From $\mu \gg m$ and the Radon-Nikodým theorem

$$
m(A)=\int_{A} f d \mu \quad(A \in \mathcal{F})
$$

where $f(x)>0$ a.e. Let $\varepsilon>0$ be any real number and choose $\delta>0$ in such a way that

$$
\delta \cdot \max \left\{\mu(X), \int f(x) d \mu\right\}<\varepsilon / 2
$$

We put $a=1+\delta$ and let

$$
E_{0}=\left\{x: \delta a^{-1} \leq f(x)<\delta\right\} .
$$

We assume that $E_{i}(n<i \leq 0)$ are chosen so that

$$
E_{i} \subset\left\{x: \delta a^{i-1} \leq f(x)<\delta a^{i}\right\} \text { for } n<i \leq 0
$$

and

$$
E_{i}^{*} \cap E_{j}^{*}=\varnothing \text { for } n<i<j \leq 0 \text {. }
$$

We put

$$
E_{n}=\left\{x: \delta a^{n-1} \leq f(x)<\delta a^{n}\right\}-\bigcup_{i=0}^{n-1} E_{i}^{*} .
$$

The sequence $\left\{E_{n}\right\}$ is inductively defined for all nonpositive integers. Further, we put $E_{-}=\bigcup_{n=0}^{\infty} E_{-n}^{*}$ and

$$
E_{1}=\{x: \delta \leq f(x)<\delta a\}-E_{-} .
$$

We assume that $E_{i}(1 \leq i<n)$ are chosen so that

$$
E_{i} \subset\left\{x: \delta a^{i-1} \leq f(x)<\delta a^{i}\right\}
$$

and

We put

$$
E_{i}^{*} \cap E_{j}^{*}=\varnothing \quad \text { for } i<j<n
$$

$$
E_{n}=\left\{x: \delta a^{n-1} \leq f(x)<\delta a^{n}\right\}-E_{-}-\bigcup_{i=1}^{n-1} E_{i}^{*} .
$$

Then $m\left(\{x: f(x)>0\}-\bigcup_{n=-\infty}^{\infty} E_{n}^{*}\right)=0$ and $E_{k}^{*} \cap E_{l}^{*}=\varnothing$ for $k \neq l$. By the Lemma we have

$$
\delta a^{n-1} \mu\left(E_{n}^{*}\right) \leq m_{E_{n}}\left(E_{n}^{*}\right) \leq \delta a^{n} \mu\left(E_{n}^{*}\right) \text { for } n=0, \pm 1, \pm 2, \ldots
$$

On the other hand, by (1) we have

$$
\sum_{n=-\infty}^{0} \delta a^{n} \mu\left(E_{n}^{*}\right) \leq \delta \mu(X)<\varepsilon / 2 .
$$

Since $f(x) \geq \delta a^{n-1}$ on $E_{n}^{*}(n \geq 1)$ by the definition, we have

$$
\int f d \mu \geq \sum_{n=1}^{\infty} \int_{E_{n}^{*}} f d \mu \geq \sum_{n=1}^{\infty} \delta a^{n-1} \mu\left(E_{n}^{*}\right) .
$$


Therefore we have

$$
\begin{aligned}
\sum_{n=-\infty}^{\infty} m_{E_{n}}(X) & =\sum_{n=-\infty}^{\infty} m_{E_{n}}\left(E_{n}^{*}\right)=\sum_{n=-\infty}^{0} m_{E_{n}}\left(E_{n}^{*}\right)+\sum_{n=1}^{\infty} m_{E_{n}}\left(E_{n}^{*}\right) \\
& <\varepsilon / 2+a \sum_{n=1}^{\infty} \delta a^{n-1} \mu\left(E_{n}^{*}\right) \quad(\text { by }(2) \text { and }(3)) \\
& <\varepsilon / 2+a \int f d \mu<\int f d \mu+\varepsilon \quad \text { (by (4) and (1)) }
\end{aligned}
$$

Consequently

$$
\sum_{n=-\infty}^{\infty} m_{E_{n}}(X)<\int f d \mu+\varepsilon
$$

Putting $E=\bigcup_{n=-\infty}^{\infty} E_{n}, m_{E}$ is a $\sigma$-finite invariant measure by Theorem 1 . The definition of $m_{E}$ and $E_{n}$, and $E_{n}^{*} \cap E=E_{n}$ imply

$$
m_{E}\left(E_{n}^{*}\right)=m_{E_{n}}\left(E_{n}^{*}\right) \text { for } n=0, \pm 1, \pm 2, \ldots
$$

From this and (5) follows

$$
m_{E}(X)=\sum_{n=-\infty}^{\infty} m_{E}\left(E_{n}^{*}\right)=\sum_{n=-\infty}^{\infty} m_{E_{n}}\left(E_{n}^{*}\right)=\sum_{n=-\infty}^{\infty} m_{E_{n}}(X)<\int f d \mu+\varepsilon
$$

Therefore $m_{E}$ is a finite invariant measure. We now show that $m_{E} \gg m$. If $m_{E}(A)=0$, then $m_{E}\left(A \cap E_{n}^{*}\right)=0$ for $n=0, \pm 1, \pm 2, \ldots$ The definition of $m_{E}$ and $E_{n}$, and $E_{n}^{*} \cap E=E_{n}$ imply $m_{E}\left(A \cap E_{n}^{*}\right)=m_{E_{n}}\left(A \cap E_{n}^{*}\right)$. By the Lemma, we have

$$
\delta a^{n-1} \mu\left(A \cap E_{n}^{*}\right) \leq m_{E}\left(A \cap E_{n}^{*}\right) \leq \delta a^{n} \mu\left(A \cap E_{n}^{*}\right),
$$

which implies $\mu\left(A \cap E_{n}^{*}\right)=0$. From $\mu \gg m$ follows $m\left(A \cap E_{n}^{*}\right)=0$ for $n=$ $0, \pm 1, \pm 2, \ldots$, hence $m(A)=0$.

Thus by Theorem 1 we obtain Theorem 3 .

3. Ergodic transformations. In this section we assume that $T$ is ergodic.

THEOREM 4. There exists a $\sigma$-finite invariant measure which is equivalent to $m$ if and only if there exists a measurable set $E$ for which $m_{E}$ is nontrivial.

REMARK. The condition is equivalent to a condition that there exists a measurable set $E$ with $m_{E}(E)>0$ (see Corollary 3 ).

COROLlARY 3. $m_{E}$ is a $\sigma$-finite invariant measure which is equivalent to $m$ if and only if $m_{E}(E)>0$.

PROOF OF THEOREM 4. By Theorem 2 the condition is necessary. We prove that the condition is sufficient. Suppose that there exists no measurable set $E$ such that $m_{E} \gg m$. That is, there exists a measurable set $A$ with $m(A)>0$ and $m_{E}(A)=0$. From this follows $m_{E}\left(A^{*}\right)=0$ and $m\left(A^{*}\right)>0$. We have $m\left(A^{* c}\right)=0$ because $T$ is ergodic, which implies $m_{E}\left(A^{* c}\right)=0$. Hence $m_{E}(X)=0$, which contradicts the existence of a nontrivial $m_{E}$. 
THEOREM 5. There exists a finite invariant measure $\mu$ with $\mu \sim m$ if and only if there exists a measurable set $E$ with $0<m_{E}(X)<\infty$.

PrOOF. By Corollary 2 the condition is necessary. Sufficiency is proved similarly in the proof of Theorem 4.

THEOREM 6. If there exists a $\sigma$-finite invariant measure $\mu$ with $\mu \sim m$, then for any $\varepsilon>0$ and any measurable set $E$ with $m(E)>0$, there exists a measurable set $F$ such that $F \subset E, m(E-F)<\varepsilon$ and $m_{F}$ is a $\sigma$-finite invariant measure with $m_{F} \sim m$.

ProOF. Let $f=d m / d \mu$. Since $f(x)>0$ a.e., for any $\varepsilon>0$ there exists $a>0$ such that

$$
m(E \cap\{x: f(x)<a\})<\varepsilon \text { and } m(E \cap\{x: f(x) \geq a\})>0 .
$$

Putting $F=E \cap\{x: f(x) \geq a\}$, we have

$$
m(E-F)<\varepsilon \text { and } m(F)>0 .
$$

Since by the definition of $m_{E}$ and $F$

$$
\begin{aligned}
m_{F}(F)=\inf \left\{\sum_{n=-\infty}^{\infty} m\left(T^{n} F_{n}\right): F=\bigcup_{n=-\infty}^{\infty} F_{n}, F_{n} \in \mathcal{F},\right. \\
\left.T^{n} F_{n} \subset F(n=0, \pm 1, \pm 2, \ldots)\right\}
\end{aligned}
$$

and by the Lemma

$$
m\left(T^{n} F_{n}\right)=\int_{T^{n} F_{n}} f d \mu \geq a \mu\left(F_{n}\right) \quad \text { for } n=0, \pm 1, \pm 2, \ldots
$$

we have

$$
m_{F}(F) \geq a \mu(F)>0,
$$

which implies $m_{F} \gg m$ by Corollary 3 .

\section{Finite invariant measures.}

THEOREM 7. Let $\mu$ and $\nu$ be finite invariant measures on $(X, \mp)$. If for any invariant measurable set $E$ we have $\mu(E)=\nu(E)$, then $\mu=\nu$.

Proof. Let $\lambda(E)=(\mu(E)+\nu(E)) / 2$ for any measurable set $E$. Then we have $\lambda \gg \mu$ and $\lambda(E)=\mu(E)$ for any invariant measurable set $E$ by assumption. By the Radon-Nikodým theorem there exists an integrable function $f$ such that

$$
\mu(E)=\int_{E} f d \lambda
$$

for any measurable set $E$. Since we have

$$
\mu(T E)=\int_{E} f(T x) d \lambda(x)
$$

and $\mu$ is invariant, we have by (6),

$$
f(T x)=f(x) \text { a.e. } \lambda \text {. }
$$


Therefore there exists an integrable function $g$ such that $g(T x)=g(x)$ for any $x$ and $g(x)=f(x)$ a.e. $\lambda$. If $f=1$ a.e. $\lambda$ does not hold, there exists $\alpha(\alpha<1$ or $\alpha>1$ ) such that an invariant measurable set

$$
F=\{x: g(x)<\alpha\} \quad \text { or } \quad G=\{x: g(X)>\alpha\}
$$

has $\lambda$-positive measure. In the former case $\mu(F) \leq \alpha \lambda(F)$, which contradicts assumption. The latter case is treated similarly.

The first half of Theorem 8 was proved by Hajian and Kakutani [3] using a Banach limit. We shall give a purely measure theoretic proof. This is also another construction of a finite invariant measure.

THEOREM 8. There exists a finite invariant measure which is equivalent to $m$ if and only if the following condition is satisfied.

(A) For any $\varepsilon>0$ there exists $\delta>0$ such that if $m(E)<\delta$, then $m\left(T^{n} E\right)<\varepsilon$ for $n=0, \pm 1, \pm 2, \ldots$.

If the condition (A) is satisfied, then for any measurable set $E$ the limit

$$
\mu(E)=\lim _{n \rightarrow \infty} \sum_{k=0}^{n-1} m\left(T^{k} E\right)
$$

exists. $\mu$ is a finite invariant measure which is equivalent to $m$.

PROOF. It is easy to prove that (A) is satisfied if there exists a finite invariant measure which is equivalent to $m$. We prove that if $(\mathrm{A})$ is satisfied, then the limit $\mu(E)$ exists and $\mu$ is a finite invariant measure which is equivalent to $m$. We put

$$
\sigma_{n}(E)=\sum_{k=0}^{n-1} m\left(T^{k} E\right)
$$

for any measurable set $E$ and a positive integer $n$. We fix a measurable set $E$. Let $\mathcal{F}_{0}$ or $\mathcal{F}_{00}$ be a $\sigma$-algebra or algebra generated by $T^{n} E(n=0, \pm 1, \pm 2, \ldots)$ respectively. $\mathcal{F}_{00}$ is countable. For any $F \in \mathcal{F}_{0}$ and for any $\varepsilon>0$, there exists $G \in \mathcal{F}_{00}$ such that

$$
m(F \cup G-F \cap G)<\varepsilon .
$$

For each $F \in \mathcal{F}_{00}$, we consider a bounded sequence $\left(\sigma_{n}(F)\right)$. Since $\mathcal{F}_{00}$ is countable, there exists a subsequence $\left(\sigma_{n_{i}}\right)$ of the sequence $\left(\sigma_{n}\right)$ of measures such that for any $F \in \mathcal{F}_{00},\left(\sigma_{n_{i}}(F)\right)$ is a Cauchy sequence. By (7) and assumption for any $F \in \mathcal{F}_{0}$, $\left(\sigma_{n_{i}}(F)\right)$ is a Cauchy sequence. We define $\tilde{\mu}$ by

$$
\tilde{\mu}(F)=\lim _{i \rightarrow \infty} \sigma_{n_{i}}(F)
$$

for any $F \in \mathcal{F}_{0} . \tilde{\mu}$ is an additive set function on $\left(X, \mathcal{F}_{0}\right)$ but by assumption $\tilde{\mu}$ is a finite measure. It is easy to check that $\tilde{\mu}$ is invariant. The nonsingularity of $T$ implies $m \gg \tilde{\mu}$. We prove that $\tilde{\mu} \gg m$. If $\tilde{\mu}(A)=0$, then $\tilde{\mu}\left(A^{*}\right)=0$. Since $\tilde{\mu}\left(A^{*}\right)=m\left(A^{*}\right)$ because $A^{*}$ is invariant, we have $m(A)=0$. Therefore the measure $\tilde{\mu}$ on $\left(X, \mathcal{F}_{0}\right)$ is finite, invariant and equivalent to $m$. If the $\operatorname{limit}_{n \rightarrow \infty} \lim _{n}(E)$ does not exist, then $\liminf _{n \rightarrow \infty} \sigma_{n}(E)<\limsup _{n \rightarrow \infty} \sigma_{n}(E)$ and therefore there exist subsequences $\left(\sigma_{p_{i}}(E)\right)$ and $\left(\sigma_{q_{i}}(E)\right)$ of $\left(\sigma_{n}(E)\right)$ converging to different limits:

$$
\lim _{i \rightarrow \infty} \sigma_{p_{i}}(E) \neq \lim _{i \rightarrow \infty} \sigma_{q_{i}}(E) .
$$


By the procedure mentioned above we obtain two different finite invariant measures $\mu$ and $\nu$ on $\left(X, \mathcal{F}_{0}\right)$ :

$$
\mu(F)=\lim _{i \rightarrow \infty} \sigma_{r_{i}}(F) \text { and } \nu(F)=\lim _{i \rightarrow \infty} \sigma_{s_{i}}(F),
$$

where $\left(r_{i}\right)$ and $\left(s_{i}\right)$ are subsequences of $\left(p_{i}\right)$ and $\left(q_{i}\right)$ respectively. For any invariant $\mathcal{F}_{0}$-measurable set $G, \mu(G)=\nu(G)=m(G)$. By Theorem $7, \mu=\nu$, which contradicts (8).

From the above for any $E \in \mathcal{\mp}$ the limit

$$
\mu(E)=\lim _{n \rightarrow \infty} \sigma_{n}(E)
$$

exists. $\mu$ is an additive set function on $(X, \mathcal{F})$ but by assumption it is a finite measure on $(X, \mathcal{F})$. It is easy to see that $\mu$ is invariant. We can prove similarly as before that $\mu$ is equivalent to $m$.

\section{REFERENCES}

1. A. P. Calderón, Sur les mesures invariantes, C. R. Acad. Sci. Paris 240 (1955), 1960-1962.

2. Y. N. Dowker, Finite and $\sigma$-finite invariant measures, Ann. of Math. (2) 54 (1951), 595-608.

3. A. B. Hajian and S. Kakutani, Weakly wandering sets and invariant measures, Trans. Amer. Math. Soc. 110 (1964), 136-151.

4. P. R. Halmos, Invariant measures, Ann. of Math. (2) 48 (1947), 735-754.

5. E. Hopf, Theory of measure and invariant integrals, Trans. Amer. Math. Soc. 34 (1932), 373393.

Department of Mathematics, Faculty of Education, Saitama University, URAWA 338, JAPAN 